\title{
Comportamento reológico de suspensões aquosas de um sistema varistor à base de $\mathrm{ZnO}$
}

\section{(Rheological properties of aqueous suspensions of a $\mathrm{ZnO}$ varistor system)}

\author{
S. L. M. Brito ${ }^{1,2}$, D. Gouvêa ${ }^{1}$, R. Ganzella ${ }^{2}$ \\ ${ }^{1}$ Laboratório de Processos Cerâmicos - Departamento de Engenharia Metalúrgica e de Materiais, Escola \\ Politécnica da Universidade de São Paulo (EPUSP), Av. Prof Mello Moraes, 2463, \\ Cidade Universitária, S. Paulo, SP 05580-900 \\ ${ }^{2}$ Indústria Eletromecânica Balestro Ltda., R. Santa Cruz, 1550 Mogi Mirim, SP 13.800-440 \\ silvio.miranda@poli.usp.br,dgouvea@usp.br
}

\begin{abstract}
Resumo
O comportamento reológico de suspensões cerâmicas apresenta forte dependência do $\mathrm{pH}$, quantidade de dispersante e concentração de sólidos. Quando dispersões são compostas por vários óxidos diferentes, o comportamento reológico pode ser afetado de maneira diferente da simples combinação dos materiais individuais. Neste trabalho, o método de viscosimetria rotacional com geometria de cilíndricos concêntricos foi utilizado para avaliar a estabilidade de suspensões cerâmicas de um sistema varistor à base de $\mathrm{ZnO}$ (aditivos: $\mathrm{Bi}_{2} \mathrm{O}_{3}, \mathrm{Sb}_{2} \mathrm{O}_{3}, \mathrm{Co}_{3} \mathrm{O}_{4}, \mathrm{Mn}_{2} \mathrm{O}_{3}, \mathrm{NiO}, \mathrm{Cr}_{2} \mathrm{O}_{3}$ ). $\mathrm{A}$ partir do comportamento reológico, foi possível estabelecer condições otimizadas de $\mathrm{pH}$ e quantidade de dispersante 9,0 e 1,70 mg/g respectivamente. Com estas condições foi determinado o limite de concentração de sólido de 43,4\%, que é pequeno e deve estar provavelmente ligado ao fator de empacotamento prejudicado pela estreita distribuição granulométrica do $\mathrm{ZnO}$.
\end{abstract}

Palavras-chave: varistor, $\mathrm{ZnO}$, reologia, estabilização eletro-estérica.

\begin{abstract}
Rheological properties of ceramic suspensions show strong dependence on $p H$, amount of dispersant and solid concentration. Most of the time, the suspensions are composed of different oxides and the rheological behavior tends to be more complex than the simple sum of single materials. In a previous work, the stability of ZnO-based varistor dispersion was analyzed regarding the physical-chemistry of the system. In this work, the method of rotational viscosimeter was used to evaluate the stability of ceramic suspensions of a ZnO-based varistor system containing $\mathrm{Bi}_{2} \mathrm{O}_{3}, \mathrm{Sb}_{2} \mathrm{O}_{3}, \mathrm{Co}_{3} \mathrm{O}_{4}, \mathrm{Mn}_{2} \mathrm{O}_{3}, \mathrm{NiO}$, and $\mathrm{Cr}_{2} \mathrm{O}_{3}$. Using this stability information, it was possible to establish optimized conditions of $\mathrm{pH}$ and amount of dispersant 9.0 and $1.70 \mathrm{mg} / \mathrm{g}$, respectively. The limit of solid concentration was determined by a simple rheological model as $43.4 \%$. The low packing value was probably due to the sharp grain size distribution of the $\mathrm{ZnO}$ powder which reduced the particle packing density.
\end{abstract}

Keywords: varistor, $\mathrm{ZnO}$, rheology, electrosteric stabilization.

\section{INTRODUÇÃO}

O processamento cerâmico envolve, em pelo menos uma de suas etapas, a obtenção de um fluido constituído por uma mistura de pós em líquidos. Estas misturas podem ser submetidas, por exemplo, a agitação, bombeamento, filtragem, moagem, conformação, tornando o controle reológico da dispersão uma ferramenta indispensável para o sucesso do processamento cerâmico.

Dentre os principais fatores que influenciam a reologia de uma dispersão cerâmica pode-se destacar: a concentração volumétrica de sólidos; as características do líquido (densidade e viscosidade), a temperatura; as características físicas das partículas (distribuição granulométrica, densidade, formadas partículas, área superficial específica), as propriedades físico-químicas da superfície dos sólidos, do tipo de interação entre as partículas (repulsão, atração); concentração de moléculas e íons presentes no líquido e da interação destas com a superfície e com o solvente [1, 2].

A mistura de óxidos para a fabricação de varistores é complexa, pois normalmente são utilizados de 5 a 7 óxidos diferentes, com características físicas e químicas principalmente do ponto de vista da superfície, o que dificulta a obtenção de uma mistura homogênea de partículas 
[3]. Como existe uma grande variedade de reações químicas no estado sólido durante a sinterização do varistor, e estas reações são responsáveis pelas fases que controlaram a microestrutura e as propriedades elétricas do dispositivo, a homogeneidade química é imprescindível para a obtenção de um varistor de alta performance.

Os varistores de $\mathrm{ZnO}$ são componentes eletro-eletrônicos aplicados à proteção de circuitos que foram inicialmente desenvolvido por Matsuoka $[4,5]$ na década de 60. Apesar de nos dias atuais serem bem conhecidos a influência de aditivos, o processamento térmico, a microestrutura e as características elétricas [6-15], existe uma carência muito grande de informações a respeito do processamento e da estabilidade desses materiais em meios aquosos, bem como de técnicas de conformação alternativas.

A aplicação destes componentes está relacionada com sistemas de proteção de sobre-tensão por apresentar característica isolante até um determinado valor de tensão (ou tensão de ruptura) a partir da qual passa a apresentar um comportamento condutor. Assim, quando existe um aumento repentino de tensão acima da tensão de ruptura parte da energia é dissipada na forma de calor protegendo o sistema de possíveis danos [16].

O objetivo deste trabalho foi o estudo das propriedades reológicas de uma mistura de óxidos de um varistor, avaliandose a influência de alguns fatores como $\mathrm{pH}$, quantidade de dispersante e concentração de sólido na estabilidade e fluidez das suspensões através de medidas da viscosidade.

\section{MATERIAIS E MÉTODOS}

O trabalho foi desenvolvido para uma formulação semelhante à desenvolvida por Matsuoka, porém adaptada pela empresa Balestro, e utilizada na produção de varistores de alta tensão conformados por prensagem para sua linha de produtos pára-raios.

As matérias-primas utilizadas neste trabalho foram $\mathrm{ZnO}$, $\mathrm{Bi}_{2} \mathrm{O}_{3}, \mathrm{Sb}_{2} \mathrm{O}_{3}, \mathrm{NiO}, \mathrm{Co}_{3} \mathrm{O}_{4}, \mathrm{Cr}_{2} \mathrm{O}_{3}, \mathrm{Mn}_{2} \mathrm{O}_{3}$, todos importados.

A análise de distribuição granulométrica do $\mathrm{ZnO}$ (óxido que prevalece na mistura varistora, normalmente utilizado em 95 a 98\% em peso) foi feita pelo método de espalhamento por laser (Malvern Instruments Ltd. série 2600).

O comportamento reológico das suspensões foi avaliado utilizando um viscosímetro do tipo RVDV-II+ (Brookfield Eng. Labs.). A técnica utilizada pelo equipamento é a de viscosimetria rotacional com geometria de cilindros concêntricos $[17,18]$ utilizando os rotores ID:SC4-18 e ID: SC4-29 em função das viscosidades medidas. O equipamento foi acoplado a um banho termostatizado, modelo TE-184 (Tecnal), para evitar oscilação térmica.

Para a obtenção das curvas de dispersão, suspensões foram preparadas em duas concentrações de sólidos diferentes: 28,7 vol.\% (70\% em peso) e 35vol.\%. (75,7\% em peso). Foram adotadasconcentraçõesiniciaisdedispersantede $\mathrm{C}_{\mathrm{L}}$ (total ativo) igual a $1,10 \mathrm{mg} / \mathrm{g}$, para que se atingisse um mínimo de fluidez que permitisse a homogeneização do sistema. As duas suspensões com volume total de aproximadamente $600 \mathrm{~mL}$, foram preparadas em um recipiente de $1000 \mathrm{~mL}$ contendo aproximadamente um quarto do seu volume preenchido com elementos de moagem de alumina, a fim de promover a desaglomeração das partículas. $\mathrm{O}$ recipiente foi mantido em moinho de bolas a $\sim 30 \mathrm{rpm}$ durante $24 \mathrm{~h}$ para desaglomeração e homogeneização do sistema. Então foram separadas 8 partes iguais de aproximadamente $150 \mathrm{~g}$ de suspensão e colocadas em potes de $100 \mathrm{~mL}$, onde foram adicionadas quantidades diferentes de dispersante e mantidas por mais $3 \mathrm{~h}$ em moinho para estabilização do sistema. A influência do tempo de envelhecimento no comportamento reológico foi feita a partir apenas da suspensão preparada com 28,7vol.\%.

A preparação e as análises das suspensões foram feitas em meio aquoso, usando água bidestilada/deionizada, $\mathrm{pH}$ $5,7 \pm 0,1$ e condutividade iônica $2 \mu \mathrm{S} / \mathrm{cm}$. Todas as medidas de viscosidade foram feitas com controle de temperatura a $25{ }^{\circ} \mathrm{C}$. As dispersões foram preparadas utilizando a mistura de óxidos e um dispersante comercial.

Para avaliar o limite de concentração de sólido, as amostras foram preparadas em recipientes de $500 \mathrm{~mL}$ contendo $1 / 4$ do seu volume preenchido por elementos de moagem de alumina. $\mathrm{O}$ volume total de amostra preparado foi aproximadamente $100 \mathrm{~mL}$ de suspensão, a quantidade de dispersante foi padronizada para $C_{L}$ igual a $1,70 \mathrm{mg} / \mathrm{g}$ para todas as amostras. Para garantir a desaglomeração e homogeneização do sistema estas amostras foram mantidas em moinho de bolas a $\sim 30 \mathrm{rpm}$ durante $24 \mathrm{~h}$.

Para avaliar a influência do $\mathrm{pH}$ sobre o comportamento reológico foi preparada uma amostra com volume total de aproximadamente $600 \mathrm{~mL}$ em um recipiente de $1000 \mathrm{~mL}$, contendo aproximadamente $1 / 4$ do seu volume preenchido com elementos de moagem de alumina, nas mesmas condições anteriores. Então, foram separadas em 8 partes iguais de aproximadamente $150 \mathrm{~g}$ de suspensão e colocadas em recipientes de $100 \mathrm{~mL}$, e ajustados os diferentes valores de $\mathrm{pH}$ a partir de solução ácida ( $\mathrm{HNO}_{3}-2 \mathrm{~N}$, Synth) e solução básica (KOH-2N, Synth). A quantidade de dispersante utilizada foi $\mathrm{C}_{\mathrm{L}}$ (total ativo) igual a $1,70 \mathrm{mg} / \mathrm{g}$, valor próximo do limite de saturação e que promove a melhor condição de dispersão no sistema. Após a adição das soluções ácidas e básicas, as amostras ficaram sob constante agitação durante $24 \mathrm{~h}$ antes de serem analisadas para garantir que a homogeneização e o equilíbrio foram atingidos.

\section{RESULTADOS E DISCUSSÃO}

A análise granulométrica do $\mathrm{ZnO}$ mostra uma distribuição bastante estreita, como pode ser visto na Fig. 1. Os diâmetros médios foram calculados utilizando médias estatísticas e são apresentados por diâmetro médio Herdan ou De Brouckere-D[4,3] igual a 1,69 $\mu \mathrm{m}$, e diâmetro médio de Sauter-D[3,2] igual a 1,68 $\mu \mathrm{m}$. O fato de se trabalhar com um pó de distribuição estreita pode ser considerado como um fator que facilita o controle da sinterização, contudo diminui o fator de empacotamento, que tem como conseqüência a impossibilidade de se obter dispersões com altas concentrações de sólidos. 


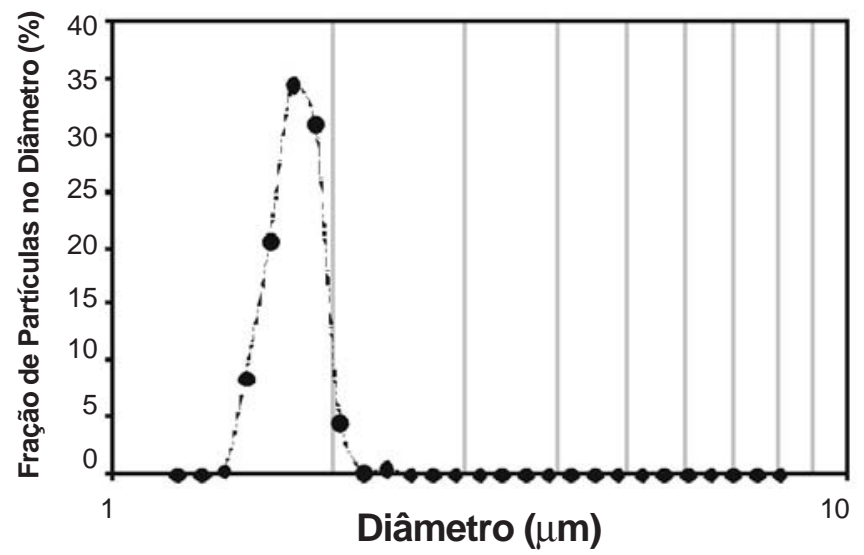

Figura 1: Distribuição granulométrica do $\mathrm{ZnO}$ obtida por espalhamento laser.

[Figure 1: ZnO particle size distribution obtained by laser scattering.]

Viscosidade em função da variação da quantidade de dispersante

A Fig. 2 mostra que a utilização de quantidades de dispersante fora dos valores considerados ideais para estabilidade do sistema (valores onde a viscosidade atinge valor mínimo) pode trazer problemas para o processamento. As amostras com diferentes quantidades de dispersante foram mantidas sob agitação durante um longo período e analisadas a seguir. Com isso, pode-se observar que o "envelhecimento" provocou mudanças na viscosidade do sistema.

A Fig. 2 sugere que quantidades abaixo e acima de valores considerados ideais (que atingem valores muito próximos do limite de saturação da superfície [19]) para desaglomeração e estabilidade do sistema não promovem um balanço de forças repulsivas e atrativas suficiente para manter o sistema desaglomerado, favorecendo a formação de aglomerados, aumentando assim a viscosidade com o passar do tempo.

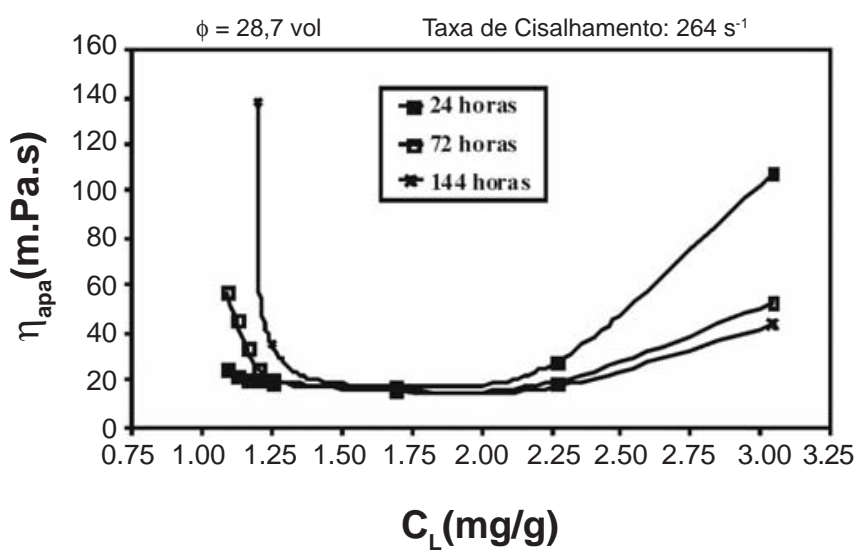

Figura 2: Viscosidade aparente em função da quantidade de dispersante $\mathrm{C}_{\mathrm{L}}$ em diferentes tempos de envelhecimento.

[Figure 2: Effects of aging of the apparent viscosity of varistor dispersion with different dispersant concentration.]

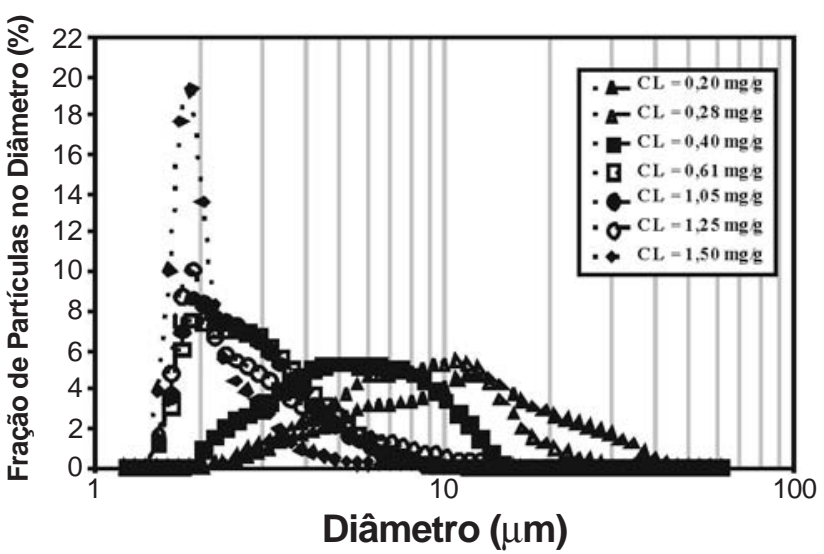

Figura 3: Distribuição granulométrica (laser) de suspensões com diferentes quantidades de dispersante.

[Figure 3: Grain sized distribution of the suspensions with dispersant different concentration.]

A Fig. 3 reforça esta hipótese mostrando o estado de aglomeração do sistema para amostras contendo diferentes quantidades de dispersante. Podem-se observar distribuições granulométricas bem largas (contendo aglomerados) para amostras com $\mathrm{C}_{\mathrm{L}}$ abaixo de aproximadamente $0,5 \mathrm{mg} / \mathrm{g}$ de dispersante, por exemplo, e desaglomeração do sistema para amostra com $\mathrm{C}_{\mathrm{L}}$ igual a $1,5 \mathrm{mg} / \mathrm{g}$.

Analisando ainda os valores de $\mathrm{pH}$ das suspensões na Fig. 4, verifica-se que o $\mathrm{pH}$ não se mantém constante com o passar do tempo.

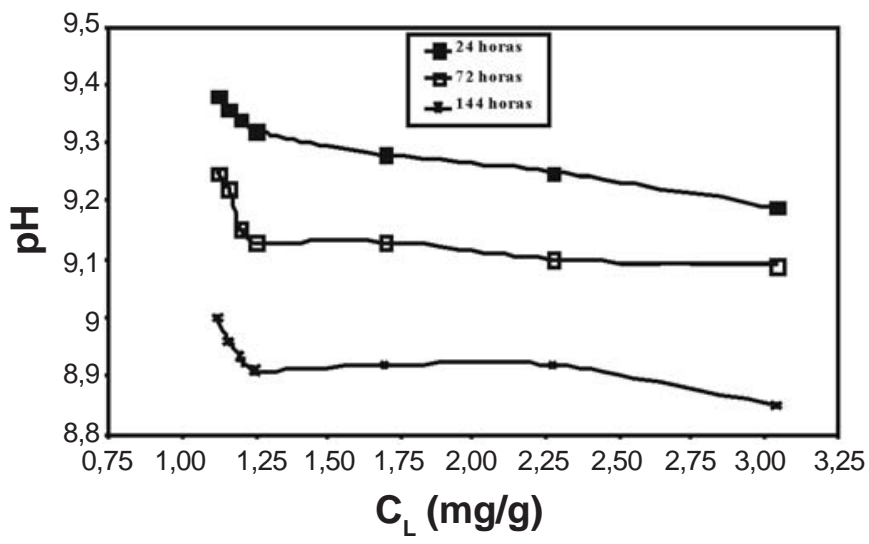

Figura 4: Valores de $\mathrm{pH}$ em função da quantidade de dispersante e do tempo.

[Figure 4: $\mathrm{pH}$ as a function of dispersant concentration for three different aging times.]

Os grupos hidroxila na superfície das partículas apresentam um caráter de dissociação podendo assumir tanto características básicas quanto ácidas devido à sua capacidade de receber ou doar um próton. Isto depende da densidade eletrônica do átomo de oxigênio presente no grupo $-\mathrm{MOH}$ (M representa um cátion metálico da superfície do sólido), podendo acontecer duas reações de dissociação distintas na presença de uma molécula de água. 
Se a densidade eletrônica for baixa, a intensidade da ligação formada com o átomo de hidrogênio polarizado é atenuada, e a reação de dissociação, mostrada na equação (A), pode ocorrer:

$$
-\mathrm{MOH}_{\text {(superficie) }}+\mathrm{H}_{2} \mathrm{O} \stackrel{\mathrm{K}_{a 1}^{s}}{\longleftrightarrow} \mathrm{MO}_{\text {(superficie) }}^{-}+\mathrm{H}_{3} \mathrm{O}^{-}
$$

Por outro lado, se a densidade eletrônica do átomo de oxigênio é elevada, a intensidade da ligação formada com o átomo de hidrogênio será intensificada e os prótons podem se tornar ligados aos grupos - $\mathrm{MOH}$, provocando uma dissociação como mostra a equação (B):

$$
-\mathrm{MOH}_{\text {(superficie) }}+\mathrm{H}_{2} \mathrm{O} \stackrel{\mathrm{K}_{\alpha 2}^{s}}{\longleftrightarrow} \mathrm{MO}_{2}^{+}{ }_{\text {(superficie) }}+\mathrm{OH}^{-}
$$

A distribuição de cargas na superfície das partículas, conforme demonstrado pelas equações (A) e (B), é afetada principalmente pelo $\mathrm{pH}$ e pela força iônica do meio. Portanto, a variação de $\mathrm{pH}$ (Fig. 4), juntamente com um forte cheiro de amônia, sugere uma possível perda de $\mathrm{NH}_{3(\mathrm{~g})}$. Com isso podemos admitir uma variação no balanço da equação (C).

$$
\mathrm{NH}_{4}^{+}+\mathrm{OH}^{-} \longleftrightarrow \mathrm{NH}_{3(\mathrm{~g})}+\mathrm{H}_{2} \mathrm{O}
$$

A variação no equilíbrio da equação (C) desencadeia um desequilíbrio no balanço de íons $\mathrm{OH}^{-}$e $\mathrm{H}^{+}$, gerando um excesso de íons $\mathrm{H}^{+}$em solução. Estes íons $\mathrm{H}^{+}$, no entanto, promovem ainda a variação no equilíbrio das equações (A) e (B), além da possível neutralização das cargas negativas das cadeias poliméricas que têm a função de manter as partículas dispersas. Estes fatores têm influência direta no grau de estabilidade da dispersão.

A Fig. 5 mostra uma curva de viscosidade aparente em função da quantidade de dispersante adicionado. $\mathrm{O}$ resultado reforça a idéia de que existe uma faixa bem definida de concentração de dispersante, onde o sistema atinge uma viscosidade mínima e

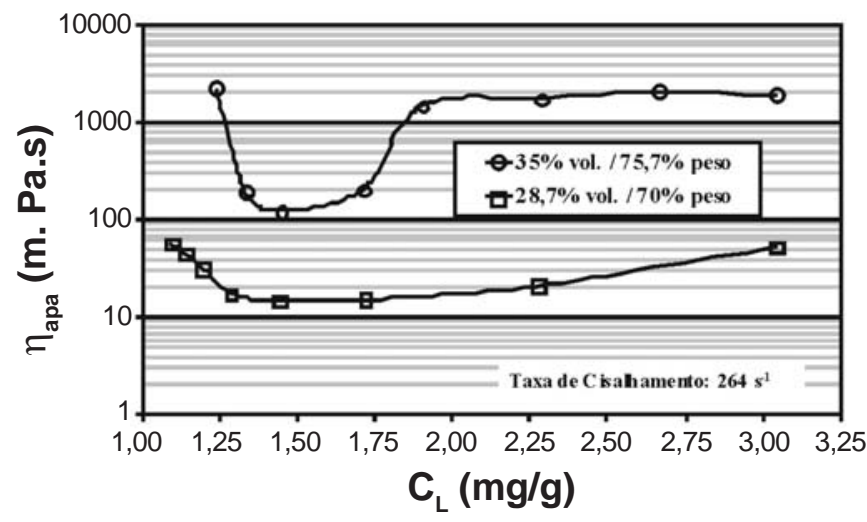

Figura 5: Viscosidade aparente em função da quantidade de dispersante $C_{L}$ (ativo) adicionado para duas concentrações de sólidos diferentes.

[Figure 5: Modification of the apparent viscosity measured at $264 \mathrm{~s}^{-1}$ for two different dispersion concentrations and increasing dispersant concentration.] nesta faixa a variação no equilíbrio das equações $(A)$ e $(B)$ e (C) tem menor influência na viscosidade.

Com base nos resultados obtidos, pode-se dizer que o intervalo de concentração de dispersante onde o sistema se encontra mais fluído e mantém seu comportamento com o envelhecimento é de 1,40 a 1,70 mg/g.

Conforme observado, o aumento da concentração de sólido leva a um aumento da viscosidade, e este aumento ainda deve reduzir a faixa de $\mathrm{pH}$ onde a viscosidade é mínima [2].

Com base no que foi apresentado e discutido nas Figs. 2 a 5 , pode-se considerar que uma quantidade de dispersante $\mathrm{C}_{\mathrm{L}}=1,7 \mathrm{mg} / \mathrm{g}$ (total ativo) é um ponto onde a viscosidade está no valor mínimo, e temos assim uma maior estabilidade do sistema.

Viscosidade em função da variação da concentração de sólidos

A viscosidade relativa de uma suspensão $\left(\eta_{\mathrm{r}}\right)$ é definida por $\eta_{a p a} / \eta_{s}$ (onde $\eta_{a p a}$ é a viscosidade aparente do fluido e $\eta_{s}$ é a viscosidade do solvente). A Fig. 6 mostra uma curva que relaciona a concentração de sólido e a viscosidade relativa para uma taxa de cisalhamento de $30 \mathrm{~s}^{-1}$. Pode-se observar que a viscosidade tende a aumentar significantemente para concentrações acima de 35\% em volume de sólidos. Para estimar a fração máxima de sólido $\left(\phi_{\mathrm{m}}\right)$ da suspensão, foi usado um modelo proposto por Liu [20], onde relacionamos a fração de sólido com a equação $1-\eta r^{-1 / 2}$, a partir desta, pode-se obter o valor teórico de $\phi_{\mathrm{m}}$ extrapolando a curva ajustada pelos valores experimentais para $1-\eta \mathrm{r}^{-1 / 2}=1$.

Na Fig. 7 pode-se observar esta extrapolação, indicando que o valor máximo teórico de sólido que pode ser obtido para uma dispersão nestas condições é de aproximadamente $\phi_{\mathrm{m}}=43,4 \%$.

Este valor pode ser considerado relativamente baixo principalmente pensando em técnicas de conformação que exigem alto grau de sólidos, como colagem de fita

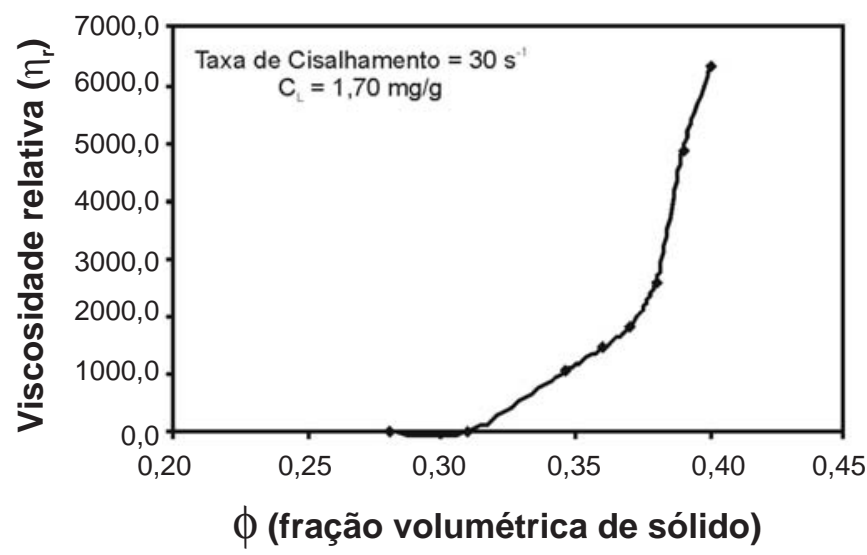

Figura 6: Viscosidade Relativa das suspensões para uma taxa de cisalhamento de $30 \mathrm{~s}^{-1}$.

[Figure 6: Relativity viscosity of the suspensions at shear rate of $30 \mathrm{~s}^{-1}$.] 


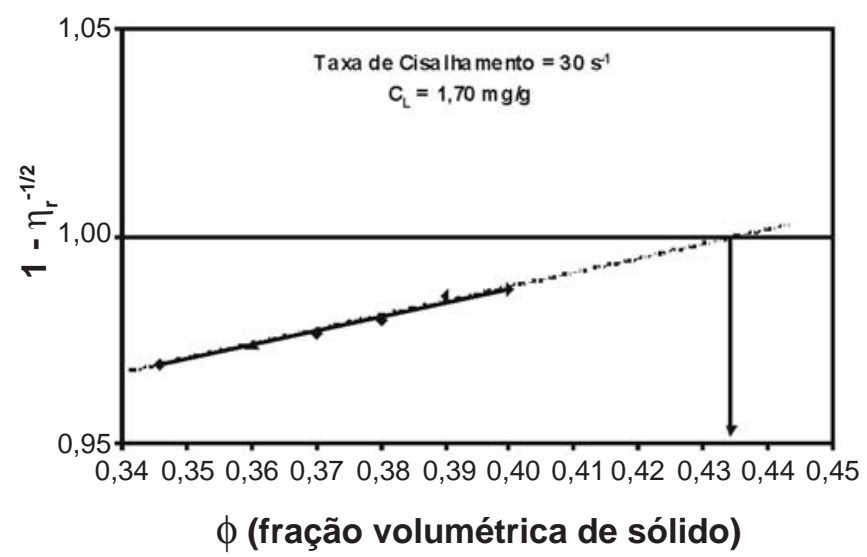

Figura 7: Determinação do limite de concentração de sólido nas dispersões pela extrapolação da curva de $1-\eta_{\mathrm{r}}^{-1 / 2}$ em função de $\phi$. [Figure 7: Maximum volume concentration determined by the extrapolation of the curve $1-\eta_{r}^{-1 / 2}$ versus $\left.\phi.\right]$

(tape casting) e extrusão. Porém, o que compromete o aumento de concentração de sólidos neste caso é o fator de empacotamento das partículas, pois trata-se de um pó de $\mathrm{ZnO}$ com distribuição granulométrica bem estreita (Fig. 1) [19], além da mistura de óxidos como o $\mathrm{Sb}_{2} \mathrm{O}_{3}$, por exemplo, que tem característica de superfície ácida [3], que é contrária ao do $\mathrm{ZnO}$ e não tem afinidade com o dispersante usado, o que dificulta muito a mistura homogênea e dispersão entre eles [21].

\section{Viscosidade em função do $\mathrm{pH}$}

Considerando o controle de viscosidade e as discussões feitas anteriormente, pode-se considerar que o pH é um dos principais fatores a ser controlado, pois age de forma determinante nas propriedades das suspensões pelo balanço das reações de equilíbrio do sistema.

A Fig. 8 confirma o que foi anteriormente proposto, mostrando que a faixa de $\mathrm{pH}$ onde o sistema pode ser

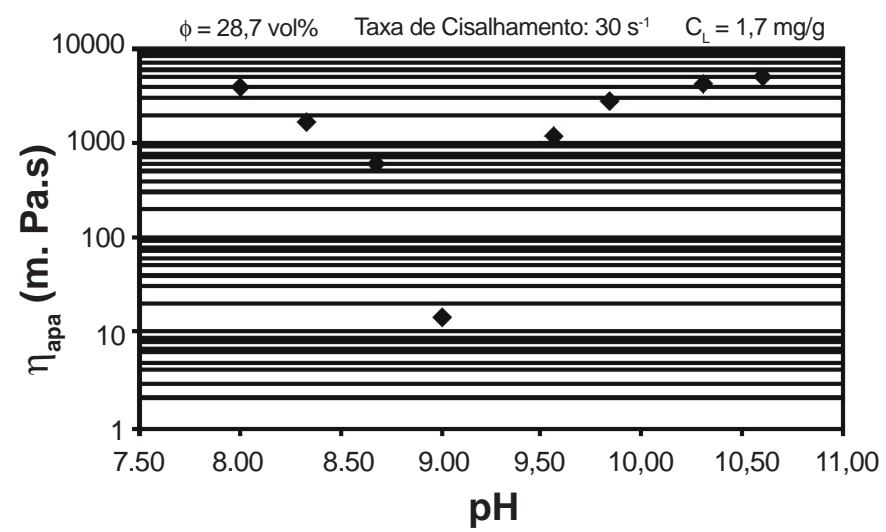

Figura 8: Viscosidade aparente em função do $\mathrm{pH}$ para uma taxa de cisalhamento de $30 \mathrm{~s}^{-1}$, fração volumétrica de $28,7 \%$ e $\mathrm{C}_{\mathrm{L}}=1,70 \mathrm{mg} / \mathrm{g}$.

[Figure 8: Apparent viscosity as a function of $\mathrm{pH}$ (shear rate $30 \mathrm{~s}^{-1}$, solid concentration of $28.7 \% \mathrm{vol}$ and $\left.C_{L}=1.70 \mathrm{mg} / \mathrm{g}\right)$.] considerado estável é muito pequena, pois qualquer variação no valor de $\mathrm{pH}$ das dispersões promove uma alteração muito grande na viscosidade do fluido. Portanto, o pH é mais uma variável do sistema que deve ter um controle minucioso.

\section{CONCLUSÕES}

O conjunto dos resultados demonstra que o $\mathrm{pH}$ e a quantidade de dispersante são duas variáveis muito importantes no controle reológico de dispersões aquosas de uma mistura para a produção de varistores à base de $\mathrm{ZnO}$.

Os valores encontrados e considerados como otimizados para estabilidade da dispersão foram $\mathrm{C}_{\mathrm{L}}=1,70 \mathrm{mg} / \mathrm{g}$ e pH=9,0. Estas condições promovem um melhor balanço nas equações de equilíbrio que prevalecem no sistema (equações (A), (B) e (C)), mantendo o sistema desaglomerado e com viscosidade mínima. Nestas condições consideradas otimizadas, pôde-se também observar que o limite de concentração de sólido nas dispersões foi aproximadamente $43,4 \%$ de sólido, que pode ser considerado baixo provavelmente pelo fato de se trabalhar com uma distribuição de tamanho de partícula estreita e também de mistura de óxidos como o $\mathrm{Sb}_{2} \mathrm{O}_{3}$, que têm característica de superfície muito diferente.

\section{AGRADECIMENTOS}

À FAPESP (Procs. 05/53241-7, 99/10798-0 e 97/061522) e à Indústria Eletromecânica Balestro Ltda.

\section{REFERÊNCIAS}

[1] I. R. Oliveira, A. R. Studart, R. G. Pillegi, V. C. Pandolfelli; Empacotamento de Partículas, Fazendo Arte Editorial, S. Paulo (2000) p. 12.

[2] F. S. Ortega, V. C. Pandolfelli, J. A. Rodrigues, D. P. F. Souza, Cerâmica 43, 280 (1997) 43.

[3] S. L. M. Brito, D. Gouvêa, R. Ganzella, Cerâmica 51, 317 (2005) 31.

[4] M. Matsuoka, T. Masuyama, Y. Lida, Jpn. J. Appl. Phys. 8 (1969) 1275.

[5] M. Matsuoka, Jpn. J. Appl. Phys. 10, 6 (1971) 736.

[6] C. Zhang, D. Zhou, W. Lu, Y. Hu, J. Mater. Sci.: Mater. Eletron. 12 (2001) 357.

[7] S. Bernik, P. Zupancic, D. Kolar, J. Eur. Ceram. Soc. 19 (1999) 709.

[8] A. T. Santhanam, T. K. Gupta, W. G. Carlson, J. Appl. Phys. 50, 2 (1979) 852.

[9] E. Olsson, I. K. L. Falk, G. E. Dunlop, R. Osterlund, J. Mater. Sci. 20 (1985) 4091.

[10] T. Takemura, M. Kobayashi, Y. Takada, K. Sato, J. Am. Ceram. Soc. 70, 4 (1987) 237.

[11] J. Han, P. Q. Mantas, A. M. R. Senos, J. Eur. Ceram. Soc. 20 (2000) 2753.

[12] S. Hindorani, D. O. Shah, M. S. Multani, J. Mater. Res. 10, 2 (1995) 461.

[13] J. P. Gambino, W. D. Kingery, G. E. Pike, L. M. Levinson, H. R. Philipp, J. Am. Ceram. Soc. 72, 4 (1989) 642. 
[14] E. Olsson, G. Dunlop, R. Osterlund, J. Am. Ceram. Soc. 76, 1 (1993) 65.

[15] T. Senda, R. Bradt, J. Am. Ceram. Soc. 74, 6 (1991) 1296.

[16] T. K. Gupta, J. Am. Ceram. Soc. 73, 7 (1990) 1817.

[17] Brookfield DV-II+ Programmable Viscometer, Operating Instr. Man. M/97-164.

[18] Brookfield Engineering Laboratories, More Solutions to Sticky Problems-A guide to getting more from your
Brookfield viscometer.

[19] S. L. M. Brito, Estudo de suspensões obtidas a partir da composição de um sistema varistor à base de $\mathrm{ZnO}$ para viabilizar técnicas de conformação como eletroforese (epd), Diss. Mestrado, Depto. Eng. Metal. Mater., EPUSP (2003). [20] Dean-Mo Liu, J. Mater. Sci. 35 (2000) 5503.

[21] R. H. R. Castro, B. B. S. Murad, D. Gouvêa, Ceram. Int. 30, 8 (2004) 2215.

(Rec. 06/09/2006, Ac. 24/11/2006) 